\title{
POTENTIAL PROPHYLACTIC EFFECT OF ORAL VERSUS INTRAARTICULAR ATORVASTATIN IN EXPERIMENTAL MODEL OF OSTEOARTHRITIS
}

\author{
$\mathscr{B}_{4}$ \\ Ali Mohamed Ali Gaballah *, Essam Ahmed Ghayaty *, \\ Nagih Rizk Mohamed * and Amany Ahmad El Hawary **, \\ Eman Abdo Elsayed Farrage *

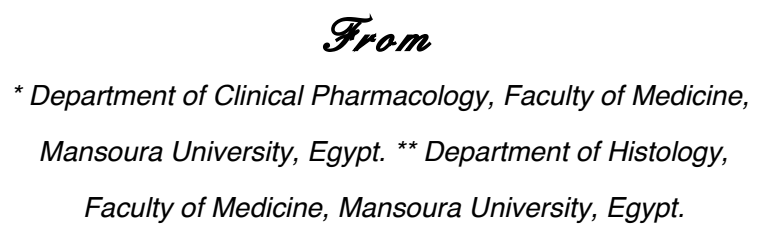

\begin{abstract}
Osteoarthritis (OA) is highly prevalent chronic disease affecting synovial joints leading to irreversible damage to all joint structures. Statins, in addition to their cholesterol-lowering activity, have pleiotropic effects. The aim of this study is to evaluate and compare the potential prophylactic effects of oral, intraarticular atorvastatin or their combination in surgically induced $\mathrm{OA}$ in rats through medial meniscal tear procedure. Rats were divided into five groups; control normal group, control non-treated osteoarthritic group, oral atorvastatine group (10 mg/kg), intraarticular atorvastatine group $(0.4 \mathrm{mg} / \mathrm{kg})$ and
\end{abstract} 145 combined oral and intraarticular atorvastatine group. The drug given immediately after surgical induction of OA for six weeks.. All atorvastatin treated groups showed significant reduction in matrix metalloproteinase 13 (MMP 13), interleukin- 1 B (IL- 1 B) and tumor necrosis factor- $\alpha$ (TNF- $\alpha$ ), malondialdehyde (MDA) and significant increase in reduced glutathione (GSH). Also, there were significant histological improvement compared to control osteoarthritic non-treated group.

It can be concluded that atorvastatin has beneficial prophylactic effect and reduced the development of MANSOURA MEDICAL JOURNAL 
$\mathrm{OA}$ lesions in a rat model of $\mathrm{OA}$ when given early after surgical induction of OA either by oral, IA or combined routs and may be suggested as a promising OA disease modifying drugs. It was also found that combined oral and IA administration of atorvastatin produced better improvement than oral or IA alone, this may be explained by synergism in action of atorvastatin when given by combined oral and IA.

Keywords: atorvastatine, Osteoarthritis, MMP13, IL1- $\beta$, MDA

\section{INTRODUCTION}

Osteoarthritis (OA) is a chronic, painful and disabling condition affecting synovial joints characterized by irreversible damage to all joint structures; articular cartilage, synovial membrane, and subchondral bone [1-2].

$\mathrm{OA}$ is a prevalent disease; $10-12 \%$ of the adult population has symptomatic $O A$ and further increase has to be expected due to the increasing prevalence of obesity and ascending life expectancy of population. OA leads to social, psychological and economic burdens in patients with substantial financial consequences [3-4]. It has a multifactorial etiology, and can be considered the product of interactions between systemic and local factors in the joint [5].

Changes to any of the joint structures can lead to an alteration in the delicate balance between the joint anabolic and catabolic processes, and consequently lead to perpetuating damage that may extend to all joint structures [6]. Local inflammatory processes within the joint accompanied with dysregulated cytokine activities as interleukin- $1 B$ (IL- 1 B) and tumor necrosis factor- $\alpha$ (TNF- $\alpha$ ) shown to contribute to pathological development of OA [7].

None of the available pharmacologic therapies till this time was proven to prevent or even retard the disease progression so end-stage in $O A$ disease may require joint replacement [8]. However, advanced researches and the progress made in the understanding of the pathophysiology of $O A$ and the identification of risk factors have guided the development of OA disease modifying drugs [9]. These drugs target to de- 
lay or even stop progression of joint tissue damage and consequently decrease the need of joint replacement.

3-hydroxy-3-methyl glutaryl coenzyme A reductase inhibitors (statins), in addition to their cholesterollowering activity, have pleiotropic effects including antiinflammatory, improvement of endothelial dysfunction, antioxidative properties, antiproliferative and antithrombotic effects [10]. Atorvastatin was chosen as it is relatively lipophilic; which can passively diffuse through different tissues including synovial membrane of the joint, has long half-life $(20-30 \mathrm{~h})$ [11] and the safety of high dose atorvastatin has been confirmed [12].

Since oral administration of statins could result in a relatively low drug bioavailability to the joints, high statin oral doses are required for beneficial effects, this may increase the risk of statin side effect, thus alternative routes of administration of the drug (transdermal, intraarticular (IA)) should be developed to establish the entire therapeutic potential of statins in clinical setting $[13,14]$.
The purpose of this study was to evaluate and compare oral and intraarticular atorvastatin and their combination in surgically induced $O A$ in rats.

\section{MATERIALS AND METHODS}

\subsection{Drugs}

- Atorvastatin as raw material (white amorphous powder, soluble in water) from Egyptian International Pharmaceutical Industry Company (EIPICO)

\subsection{Animals}

Fifty male Sprague-Dawley rats weighing 250-300 grams each were obtained from the medical experimental research center (Mansoura faculty of medicine). Rats were divided into five groups $(n=10 /$ group) and housed under standard conditions for at least 1 week to acclimate before starting the experiments. They were put in similar optimum housing condition with free access to food and water. Animals were kept in cages at a room with controlled temperature $26{ }^{\circ} \mathrm{C}$ and on a 12-h light-dark cycle. The local animal ethics committee has approved in all experimental procedures.

MANSOURA MEDICAL JOURNAL 


\subsection{Induction of osteoarthritis}

Surgical induction of OA was performed on forty rats (four groups). Animals were anesthetized by using mixture of diazepam $(5 \mathrm{mg} / \mathrm{kg})$ and ketamin $(50 \mathrm{mg} / \mathrm{kg})$ intraperitoneal, anesthesia maintained with $2 \%$ isoflurane inhalation.

The right knee of the rats was shaved, sterilized by bitadine solution, then the medial collateral ligament was exposed through a medial midline skin incision, and was transected and the medial meniscus is reflected medially toward the femur and then cut. Skin incisions were closed with5-0 Vicryl sutures, and animals were allowed to recover from anesthesia $[15,16]$.

\subsection{Animal Grouping and Ex-} perimental Design

Group 1: SHAM rats that exposed to the same surgical procedure but without cutting the medial medial collateral ligament and the medial meniscus unoperated. They recieved normal saline orally once daily for six weeks.

The forty rats with surgical induction of osteoarthritis were divided Vol. 44, No. 1 \& 2 Jan. \& April, 2015 into four group of 10 rats in each, as a following:

Group2: non-treated osteoarthritic rats. They received oral normal saline once daily from the first day of surgery for six weeks.

Group 3: osteoarthritic rats were treated with atorvastatin solution orally at a dose of $10 \mathrm{mg} / \mathrm{kg} /$ day and administrated by a gastric gavage from 1st day of surgery for six weeks [17].

Group 4: osteoarthritic rats were treated with atorvastatin solution intraarticularly at a dose of $0.4 \mathrm{mg} / / \mathrm{kg}$ and injected intraarticularly by insulin syringe once a week from 1st day of surgery for six weeks [18]

Group 5: osteoarthritic rats were treated with combined oral atorvastatin $10 \mathrm{mg} / \mathrm{kg} /$ day and intraarticular atorvastatin $0.4 \mathrm{mg} / \mathrm{kg} /$ from $1 \mathrm{st}$ day of surgery once a week for six weeks

At end of study rats were sacrificed using an over-dose of thiopental sodium $(75 \mathrm{mg} / \mathrm{kg}$ body weight, i.p) [19]. Blood was withdrawn from the heart of rats by $5 \mathrm{ml}$ syringe. 
Blood samples were collected in a dry test tubes permitted to clump for 30 minutes prior to centrifuge at 4000 revolutions per minute for 15 minute and then serum was separated and collected in Eppendorf tube and kept frozen at $-20^{\circ} \mathrm{C}$ till the time of measurement of MMP13, IL-1 $\beta$, TNF- $\alpha$, MDA. Other amount of blood (for measurement of GSH) was collected using heparin as an anticoagulant. Then the blood was diluted 4 times by distilled water to lyse the erythrocyte and the supernatate was collected (erythrocyte lysate).

\subsection{Biochemical Assay}

1- Determination of serum MMP 13 using rat MMP 13 ELISA Kit (Eiaab science com, Catalog No E0099r)

2- Determination of sera inflammatory markers:

A. IL-1 $\beta$ concentration using rat IL-1 $\beta$ ELISA Kit (Boster biological technology, Catalog No. EK0393)

B. TNF- $\alpha$ concentration using rat TNF- $\alpha$ ELISA Kit (Rat TNF- $\alpha$ ELISA Kit, RayBio.USA)

\section{4- Oxidative stress markers:}

A. Colorimetric determination of reduced glutathione (GSH) in fresh heparinized blood according to Beutler et al. [20] using (Bio-diagnostic kit. Egypt)

B. Colorimetric determination of serum lipid peroxide (Malondialdehyde) according to Ohkawa et al. [21] using (Bio-diagnostic kit. Egypt)

\subsection{Histopathological Examination}

After extraction of the skin, osteotomy above and under the knee joint and fixation of the specimens in $10 \%$ buffered formalin $24 \mathrm{~h}$ were done [22]. The specimens then decalcified in $10 \%$ nitric acid for 5 days. Paraffin wax tissue blocks were prepared for sectioning by sledge microtome at $4 \mu \mathrm{m}$ thickness. The obtained tissue sections were collected deparaffinized on glass slides. Histological examination of the slides stained by Haematoxyline and Eosin $(H / E)$, and semi quantitative histological lesions grading (0-5) performed with scoring system in accordance to Khan et al. [23] as a follow:

0 : Normal cartilage: Intact surface layer, normal chondrocytes, normal morphology of cells and continuous matrix.

1: Rough surface: Irregular superficial layer (0.25), condensation of

MANSOURA MEDICAL JOURNAL 
superficial layer (0.25), proliferation of chondrocytes $(0.25)$ and initiation of cluster formation (0.25).

2: Surface discontinuity: All above and discontinuous superficial layer (1.20), cell death till mid zone (1.20), hypercellularity (1.20), disorientation of chondrocyte columns (1.20) and cluster formation (1.20).

3: Cleft formation: All above and large patches of cell death (2.25), sloughing of layers till mid zone (2.25), mild loss of matrix (2.25) and hypertrophy (2.25).

4: Erosion: Sloughing of cellular layers (3.20), maximum loss of matrix (3.20), clusters of chondrocytes (3.20), hypocellularity (3.20) and multiple patches of cell death (3.20).

5: Denudation: Denudation of bone (4.25), maximum loss of cellular layers and matrix (4.25), micro fracture (4.25) and initiation of fibrosis (4.25).

\subsection{Statistical analysis}

Data were analyzed using Statistical package for social science (SPSS) version 21. In the statistical comparison between the different Vol. 44, No. 1 \& 2 Jan. \& April, 2015 groups, the significance of difference was tested using ANOVA (analysis of variance): Used to compare between more than two groups of numerical (parametric) data followed by post-hoc tukey for multiple comparisons. A P value $<0.05$ was considered statistically significant in all analyses.

\section{RESULTS}

3.1 Effect of atorvastatin on MMP-13

Surgical induction of $O A$ in rats produced highly significant increase in serum MMP13 in control osteoarthritic group (group 2) compared to control non-arthritic rats (group 1).

Oral, IA and combined (groups 3, 4, 5 respectively) showed highly significant decrease in MMP-13 as compared with control OA group but still significantly higher than control normal group except group 5 which receive combined treatment where MMP-13 level not significantly differ from control non-arthritic group. There was no significant difference between oral and IA treated groups while combined group showed significant decrease in MMP13 compared with either oral or IA prophylactic group. 
3.2 Effect of atorvastatin on inflammatory markers

Surgical induction of $O A$ in rats produced significant increase in serum IL-1 $\beta$, TNF- $\alpha$ in control osteoarthritic group as compared with control non-osteoarthritic rats Oral, IA or combined (groups 3, 4, 5 respectively) produced highly significant decrease in IL-1 $\beta$ and TNF- $\alpha$ compared with control OA group. Group 5, which receive combined oral and IA atorvastatin, IL-1 $\beta$ and TNF- $\alpha$ levels not significantly differed from control non-arthritic group. There was no significant difference between oral and IA treated groups while combined group showed significant decrease in IL-1 $\beta$ and TNF- $\alpha$ compared with either oral or IA treated group alone (Table 2).

3.3 Effect of atorvastatin on oxidative stress markers

Surgical induction of OA produced a significant decrease in $\mathrm{GSH}$ and increase in MDA as compared to normal group. Oral, IA, combined (groups 3, 4, 5 respectively) produced significant increase in $\mathrm{GSH}$ level and decrease in MDA compared with control OA group, Also, there was no significant difference between oral and IA groups while combined group showed significant increase in GSH and significant decrease in MDA compared with either oral or IA prophylactic group alone. Combined oral and IA atorvastatin treated group in both markers not significantly differ from control nonarthritic group (Table 3).

3.4 Effect of atorvastatin on histopathological score

As compared to histopathological examination of the normal non arthritic rats (figure 1) which showed intact histological picture; intact surface layer, normal chondrocytes orientation, morphology of cells and continuous matrix (Grade 0), the histopathological examination of the osteoarthritic rats (figure 2A, 2B) showed loss of superficial layer, cleft formation, cell death, hypocellularity, maximum loss of cellular layers and matrix, erosion and matrix loss and initiation of fibrosis (Grade 5)

The histopathological examination of the treated groups showed attenuation in the degree and severity of pathological changes compared to osteoarthritic non-treated group (figures $3,4,5$ respectively).

MANSOURA MEDICAL JOURNAL 
Surgical induction of $O A$ in rats produced highly significant increase in histopathological score in control osteoarthritic group (12.76 \pm 1.35$)$ (Table 4) as compared with that of control non-osteoarthritic rats (group 1) $(0 \pm 0)$. Oral, IA and combined groups showed highly significant improvement in histological scores $(3.28 \pm 0.51,2.95 \pm 0.44,0.835 \pm 0.98)$ compared with OA group but still sig- nificantly higher than control normal group except group 5, which receive combined treatment, which show non-significant difference from control non-arthritic group. Combined group showed significant improvement compared with either oral group alone or IA group alone while there was no significant between oral and IA groups

Table 1: effect of atorvastatin on MMP 13 level

\begin{tabular}{|c|c|}
\hline Animal groups $(\mathrm{n}=\mathbf{1 0})$ & $\begin{array}{l}\text { MMP13 }(\mathrm{pg} / \mathrm{ml}) \\
\text { Mean } \pm \text { SD }\end{array}$ \\
\hline Group 1: control non osteoarthritic group & $6.15 \pm 2.58$ \\
\hline Group 2: control osteoarthritic group & $20.77 \pm 3.30$ \\
\hline & $\mathrm{P} 1 *$ \\
\hline Group 3: Oral treated group & $\begin{array}{c}13.25 \pm 1.90 \\
\text { P1* } \\
\text { P2 }{ }^{*}\end{array}$ \\
\hline Group 4: IA treated group & $\begin{array}{c}12.88 \pm 2.64 \\
\text { P1* } \\
\text { P } 2 * \\
\text { P } 3 \text { NS }\end{array}$ \\
\hline Group 5: combined oral and IA group & $\begin{array}{c}8.05 \pm 0.54 \\
\text { P1 NS } \\
\text { P 2 * } \\
\text { P3 * } \\
\text { P4 * }\end{array}$ \\
\hline
\end{tabular}

SD: standard deviation

$*$ : significant $(p \leq 0.05)$

NS: non-significant $(p>0.05)$

P1: significance with control non-osteoarthritic group

P2: significance with control osteoarthritic group

P3: significance with oral treated group

P4: significance with combined oral and IA group 
Table 2: effect of atorvastatin on inflammatory markers

\begin{tabular}{|c|c|c|}
\hline Animal groups $(\mathrm{n}=10)$ & $\underset{\text { Mean }}{\text { I L1- SD }}\left(\begin{array}{l}(p g / m l) \\
\text { SD }\end{array}\right.$ & $\begin{array}{l}\text { TNF- }(p g / m l) \\
\text { Mean } \pm \text { SD }\end{array}$ \\
\hline Group 1: control non-osteoarthritic group & $39.45 \pm 5.56$ & $10.51 \pm 1.51$ \\
\hline Group 2: control osteoarthritic group & $\begin{array}{c}92.27 \pm 12.25 \\
\mathrm{P} 1 *\end{array}$ & $\begin{array}{c}22.61 \pm 3.32 \\
\mathrm{P} 1 *\end{array}$ \\
\hline Group 3: oral treated group & $\begin{array}{c}53.34 \pm 5.67 \\
\mathrm{P} 1 * \\
\mathrm{P} 2 *\end{array}$ & $\begin{array}{c}15.10 \pm 0.55 \\
\mathrm{P} 1 * \\
\mathrm{P} 2 *\end{array}$ \\
\hline Group 4: IA treated group & $\begin{array}{c}50.82 \pm 2.98 \\
\text { P } 1^{*} \\
\text { P } 2 * \\
\text { P } 3 \text { NS }\end{array}$ & $\begin{array}{c}15.04 \pm 1.66 \\
\text { P }{ }^{*} \\
\text { P } 2 * \\
\text { P } 3 \text { NS }\end{array}$ \\
\hline Group 5: Combind oral and IA treated gro & $\begin{array}{c}39.74 \pm 6.03 \\
\text { P1 NS } \\
\text { P 2* } \\
\text { P3 * } \\
\text { P4 * }\end{array}$ & $\begin{array}{c}11.68 \pm 1.33 \\
\text { P1 NS } \\
\text { P 2* } \\
\text { P3 * } \\
\text { P4 * }\end{array}$ \\
\hline
\end{tabular}

standard deviation

*: significant $(p \leq 0.05)$

NS: non-significant $(p>0.05)$

P1: significance with control non-osteoarthritic group

P2: significance with control osteoarthritic group

P3: significance with oral treated group

P4: significance with combined oral and IA group

Table 3: effect of atorvastatin on oxidative stress markers

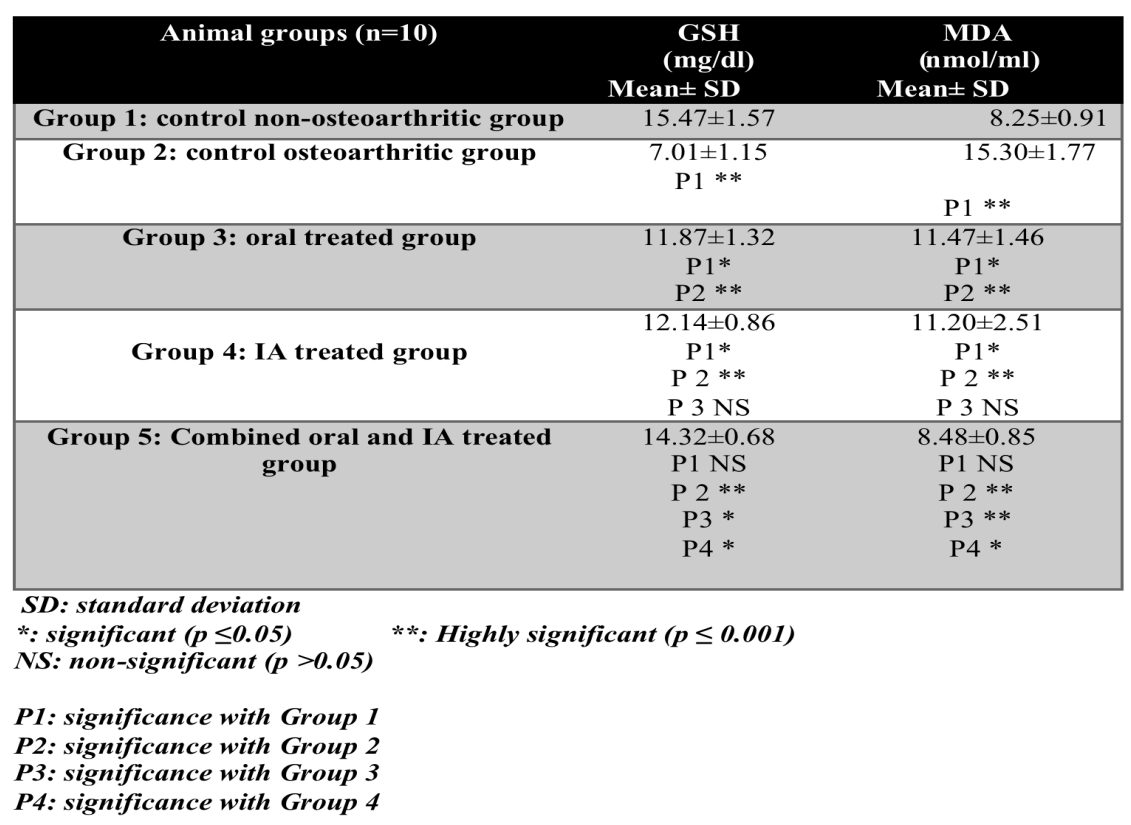

MANSOURA MEDICAL JOURNAL 
Table 4: effect of atorvastatin on histological score

\begin{tabular}{|cc|}
\hline Animal groups $(\mathbf{n}=\mathbf{1 0})$ & $\begin{array}{c}\text { histopathological score } \\
\text { Mean }\end{array}$ \\
\hline Group 1: control non-osteoarthritic & $\mathrm{O} \pm 0$ \\
\hline Group 2: control osteoarthritic group & $12.76 \pm 1.35$ \\
\hline Group 3: Oral treated group & $\mathrm{P} 1 *$ \\
\hline Group 4: IA treated group & $3.28 \pm 0.51$ \\
& $\mathrm{P} 1 *$ \\
& $\mathrm{P} 2 *$ \\
\hline Group 5: combined oral and IA group & $2.95 \pm 0.44$ \\
& $\mathrm{P} 1 *$ \\
& $\mathrm{P} 3 *$ \\
& $0.835 \pm 0.98$ \\
\hline & $\mathrm{P} 1 \mathrm{NS}$ \\
& $\mathrm{P} 2 *$ \\
& $\mathrm{P} 3 *$ \\
\hline
\end{tabular}

SD: standard deviation

*: significant $(p \leq 0.05)$

NS: non-significant $(p>0.05)$

P1: significance with Group 1 P2: significance with Group 2 P3: significance with Group 3 P4: significance with Group 4

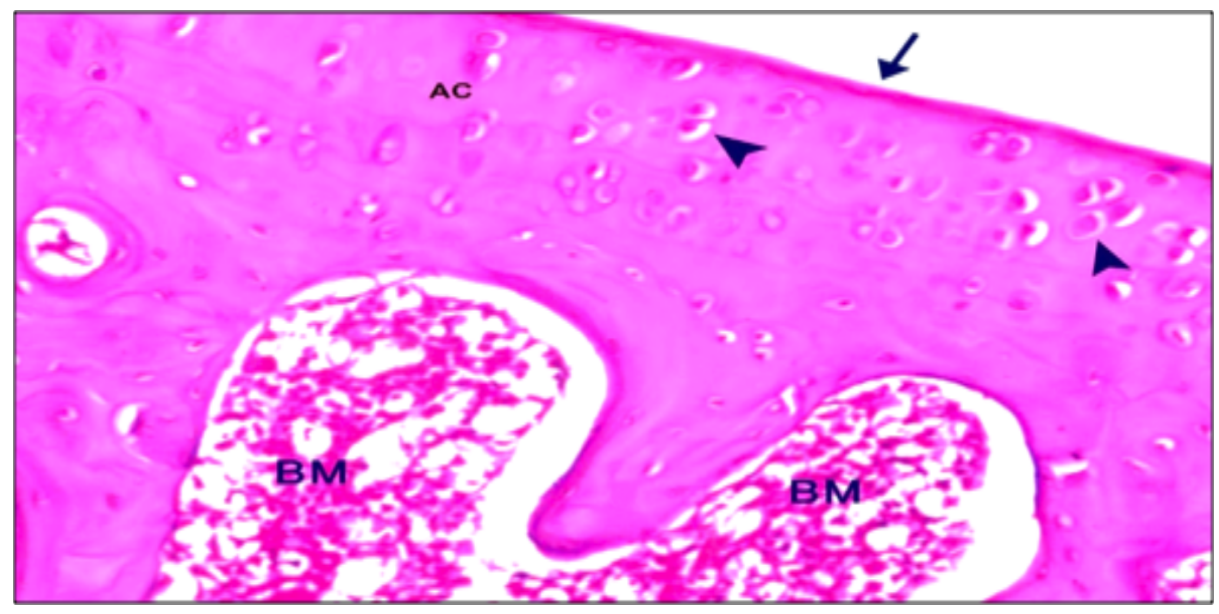

Figure (1): Histopathological findings of hematoxylin and eosin-stained normal articular cartilage section of proximal tibia: The articular cartilage shows (AC) smooth surface (arrow). The chondrocytes appear normal in the matrix (arrow heads). Normal bone marrow (BM) are seen.

Vol. 44, No. 1 \& 2 Jan. \& April, 2015 


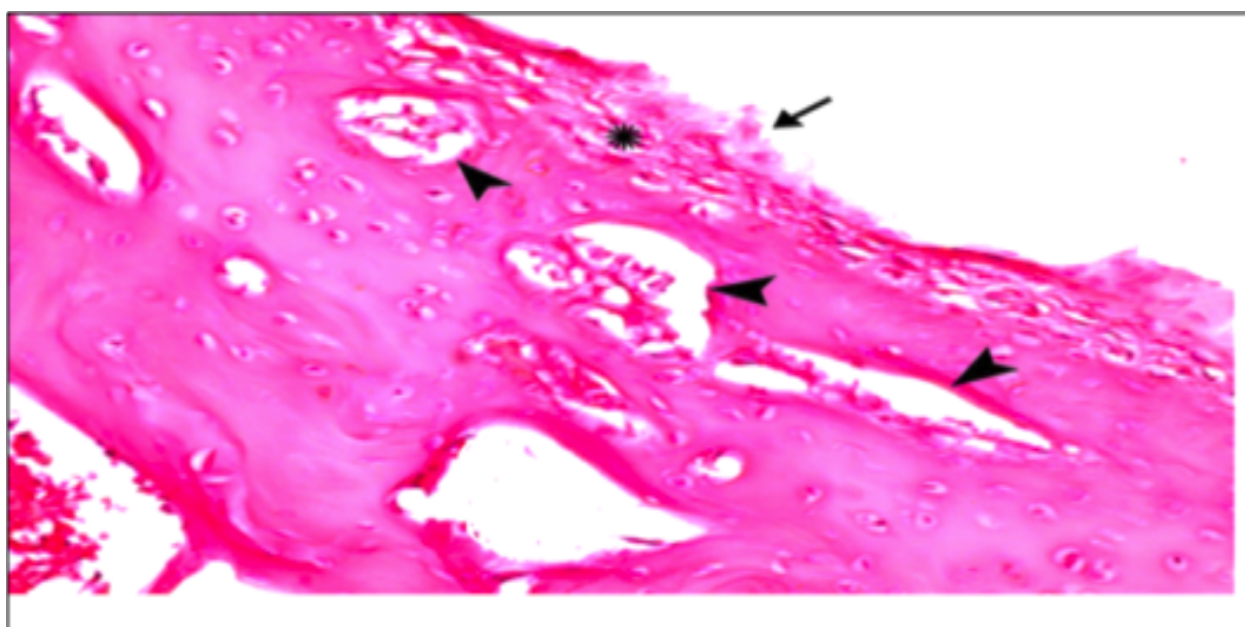

Figure (2 A): Histopathological findings of hematoxylin and eosin-stained osteoarthritic articular cartilage section: The cartilage from OA group shows erosion in surface of the articular cartilage (arrow) with the presence of underlying fibrous tissue (asterisk). Abnormal cavities are seen in matrix (arrow heads) .

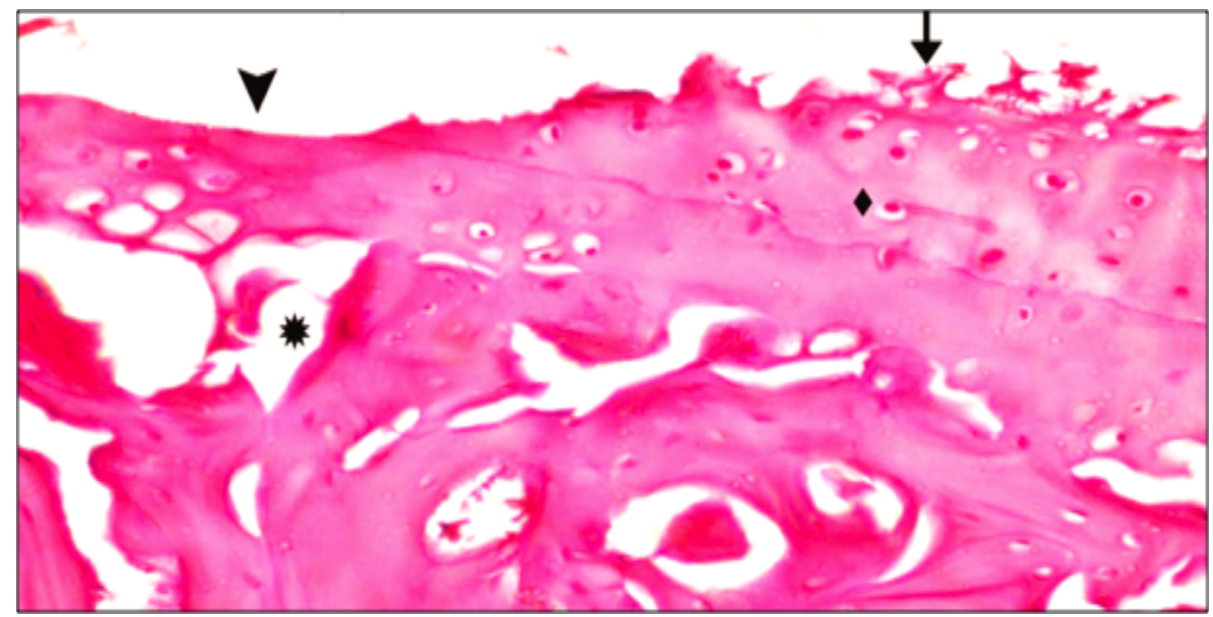

Figure (2 B): Histopathological findings of hematoxylin and eosin-stained osteoarthritic articular cartilage section: The cartilage from OA group shows disrupted articular cartilage surface (arrow), area of ulceration (arrow head) and decrease number of chondrocytes (diamond) and some cell death. There are subchondral bone cavities (asterisk). 


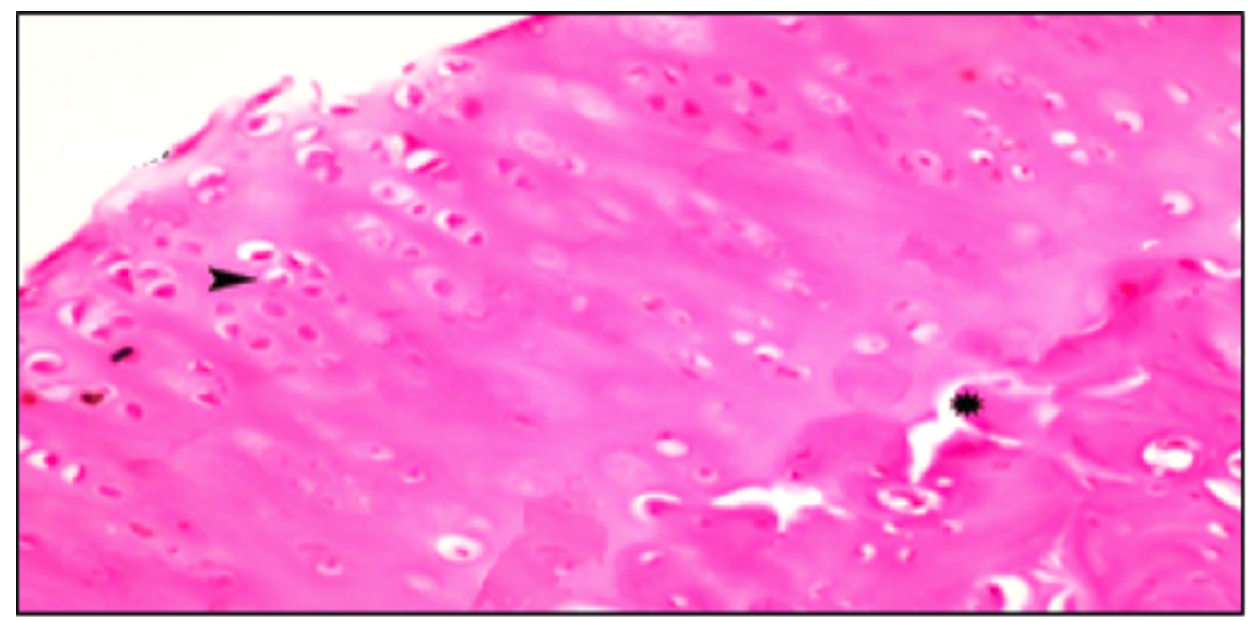

Figure (3): Histopathological findings of hematoxylin and eosin-stained oral atorvastatin treated group: The cartilage from oral group shows less disrupted surface of articular cartilage, apperantly increase articular cartilage thickness with increased number of chondrocytes (arrow head). Small cysts seen in subchondral bone (asterisk)

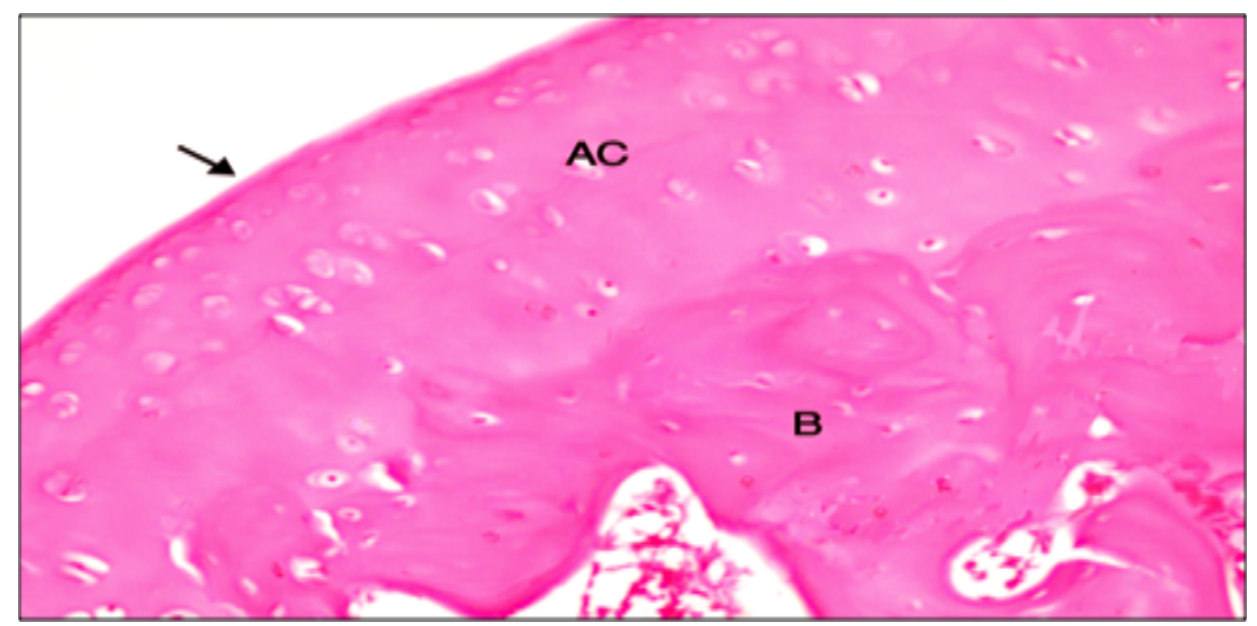

Figure (4): Histopathological findings of hematoxylin and eosin-stained IA treated group: The cartilage from IA group shows smooth surface of articular cartilage (arrow), slight increase in articular cartilage thickness with nearly normal number of chondrocytes .

Vol. 44, No. 1 \& 2 Jan. \& April, 2015 


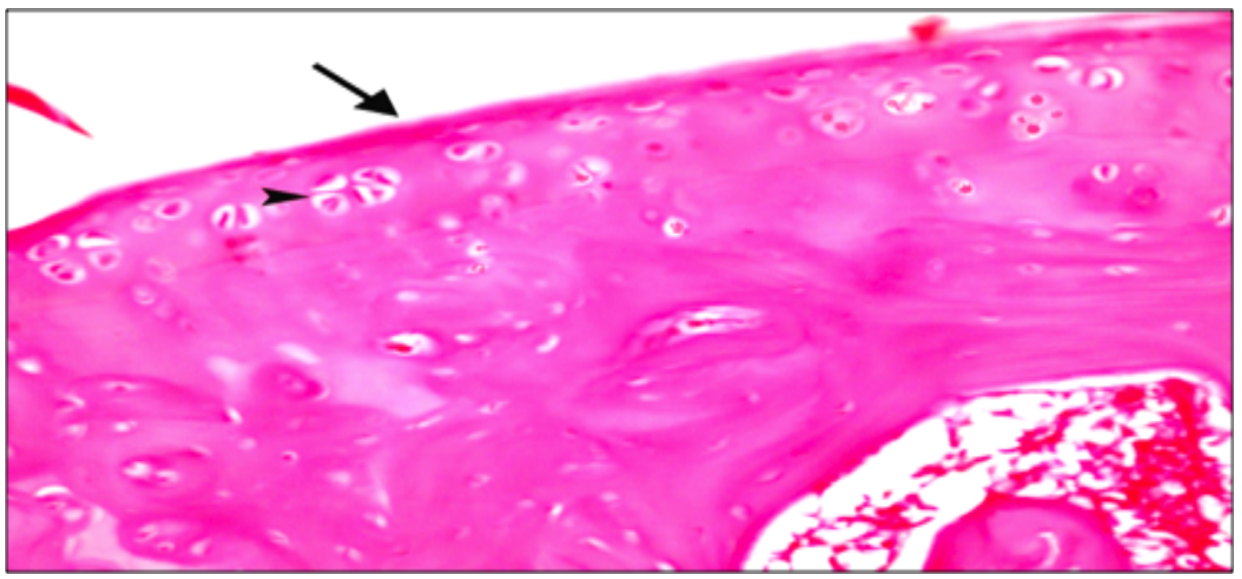

Figure (5): Histopathological findings of hematoxylin and eosin-stained of combined oral and IA group: The cartilage from combined group showing smooth surface of articular cartilage (arrow), nearly normal articular cartilage thickness with nearly normal number of chondrocytes (arrow head).

\section{DISCUSSION}

According to American College of Rheumatology, the pharmacologic recommendations for initial management of OA primarily aims to alleviate symptoms, reduce pain and control inflammation with paracetamol, NSAIDs, opioid analgesic or intraarticular corticosteroid injections [24].

Current drug development focuses on a new strategy in treatment of $O A$ to modify the progression of joint structural changes and causes either slowing the rate of disease progression, a complete stop of disease pro- gression or even reverse the disease [25].

Surgically induced OA models may be more clinically relevant than chemically induced models because the histopathological and biochemical changes that occur following surgical induction of $O A$ are more similar to those seen in human OA [15].

In the present study, we evaluated the possible effect of statin in an experimental model of osteoarthritis as a potential $O A$ disease modifying drug. Atorvastatin was given as oral (10 mg/kg/day), IA (0.4mg/ $/ \mathrm{kg} /$ week) or by both oral and IA starting from

MANSOURA MEDICAL JOURNAL 
first day of surgical induction of $O A$ for successive 6 weeks.

The disease was evaluated by histopathology and by chemical markers that affect the pathogeneses of the joint structural changes e.g pro-inflammatory cytokines (IL$1 \beta$ and $T N F \alpha$ ), proteolytic enzymes (MMP-13) and oxidative stress markers (MDA and GSH).

After induction of $\mathrm{OA}$ in rat, it was found that there was significant increase of MMP-13 in osteoarthritic group compared to normal nonarthritic group. The increase in level of MMP-13 came in agreement with other previous studies as Akasaki et al, 2009 [26]. MMPs are the proteolytic enzymes increased in $O A$ and play a central role in the degradation of articular cartilage. MMP-13 is the key of these enzymes as it is the most proficient at hydrolyzing bonds in type II collagen, the most abundant type of collagen in articular cartilage [27, 28]. Atorvastatin treatment in this study produced significant reduction of MMP-13in all atorvastatin treated groups compared to nontreated arthritic group.

It was found also that there is sig- nificant increase of IL-1 $\beta$ and TNF $\alpha$ in osteoarthritic group as compared to normal non-arthritic group. The progression of cartilage degradation is mediated largely by proinflammatory cytokines mostly IL-1 $\beta$ and TNF $\alpha$. These cytokines contribute to tissue destruction by disrupting the balance of the catabolic and anabolic activities of chondrocytes. Much of the activities of these proinflammatory cytokines are mediated by the activation of transcription factor NF?B. As a result, OA chondrocytes reduce expression of extracellular matrix components as type II collagen and aggrecan as well as increased production of proteolytic enzymes as MMPs [29] Atorvastatin produced significant reduction of IL-1 $\beta$ and TNF $\alpha$ in all treated compared to OA group

These anti-inflammatory effects of statins appeared to be independent of the cholesterol lowering activity. Statin inhibit mevalonate synthesis leading to a reduction in the synthesis of important intermediates, such as the isoprenoids geranylgeranylpyrophosphate (GGPP) and farnesyl-pyrophosphate (FPP), These intermediates are involved in the posttranslational prenylation of several 
Ali Mohamed Ali Gaballah et al ....

proteins (e.g., Ras, Rho, Rac) that modify a variety of cellular processes including cellular signaling, differentiation, proliferationcoagulation, oxidation, inflammatory and immunologic responses [30]. Statins through its inhibition of these intermediate have important pleiotropic effects; improvement of endothelial dysfunction, antioxidative properties, antiinflammatory, antiproliferative and antithrombotic effects and neoangiogenesis so statins may be of potential therapeutic use in a variety of non-vascular diseases, including autoimmune diseases, multiple sclerosis, arthritis, dementia and some types of cancer [10]

Statins likely inhibit the expression of multiple inflammatory cytokines through its ability to inhibit nuclear factor kappa B (NF-кB) activation that is transcriptional regulator of more than 40 inflammatory genes. NF-KB is known to be activated via Rho GTPases and statin inhibit NF-kB through down-regulation of Rho-related protein activation. Statins also activate antiinflammatory transcription factors known as peroxisome proliferator-activated receptors, intracellular ligand-activated transcription factors that interfere with NF-KB transcriptional activity [31].

The induction of OA was associated with increase in MDA, NO and decrease in reduced glutathione as compared to non-arthritic control group in the current study.

Osteoarthritis is characterized by increased markers of oxidative stress; human articular chondrocyte can actively produce reactive oxygen species (ROS). ROS with oxidative activity play an important role in the chondrocyte catabolic program being the mediators and effecters of cartilage damage [32] This negative effects through damage of membranes, modification of proteins and DNA which disturb cartilage anabolism and promote catabolism and induce cell death [33].

Atorvastatin treatment produced marked improvement of oxidative stress markers, it decreases MDA while GSH was increased in all treated groups

Statins reduce the production of ROS by inhibiting the assembly and activation of the nicotinamide adenine dinucleotide phosphate

MANSOURA MEDICAL JOURNAL 
(NADPH) complex. In addition, they reduce oxidative stress by controlling nitric oxide production and possibly through their anti-inflammatory effects [34]. These effects of statin result from inhibition of mevalonate pathway which results in a reduction in the synthesis of important intermediates including isoprenoids [35].

Histological studies are more precise to detect the accurate severity of OA. A simple and reproducible histopathological score of experimentally induced $\mathrm{OA}$ in animals provide the necessary information about structural changes in joints and their degree of severity [23]. In the present study, histopathological examination and histological scoring were done according to Khan et al, 2013 [23]. Development of OA in control arthritic rats was evident in histopathological examination as there were loss of superficial layer, cleft formation, cell death, hypocellularity, maximum loss of cells and matrix, erosion and matrix loss and fibrosis recording score of 12.8 as compared the normal control saline treated non arthritic rats where histological picture is completely normal; intact surface layer, normal chondrocytes orientation, morphology of cells and contin- uous matrix and allocated as zero score. Groups treated with atorvastatin showed significant histological improvement than non- treated arthritic group.

The combined oral and IA group showed significant improvement than either oral or IA alone. This can be explained by synergistic effect of combined oral and IA. The oral administration of statins result in a relatively low drug bioavailability to the joints, and to reach with sufficient amount high statin doses are required which increase the risk of statin-associated side effects [14]. Oral administration of statin in usual dose improve OA mainly through its systemic effect. It has been proposed that the progression of $\mathrm{OA}$ to advanced states is driven by atheromatous vascular disease leading to subchondral bone ischaemia and contributes to joint cartilage degeneration as a result of disturbance of its nutritional supply [36]

Statins prevent atherosclerosis by their lipid-lowering properties and antioxidant effects as they reduce susceptibility of LDL to oxidation and reduce the amount of oxidized Lowdensity lipoprotein (LDL) in plasma 
and within the atherosclerotic lesions [37]. statins also modulate coagulation and exert antithrombotic effects via a number of mechanisms including inhibition tissue factor synthesis and increasing thrombomodulin formation via inhibition of geranylgeranylation of the Rho/Rho kinase pathway and, in turn nuclear factor-?B activity, increasing endothelial nitric oxide synthase activity, enhance fibrinolysis by its effect of protein $C$ and inhibit factor $\mathrm{V}$ and XIII activation [38].

On the other hand, IA atorvastatin improve OA mainly through its local effect on the cartilage and subchondral bone more than in oral rout. Statin has direct anabolic effects on cartilage as it enhances aggrecan and type II collagen gene expression and proteoglycan synthesis. Such an effect appeared to be mediated by the concomitant up-regulation of bone morphogeneic potein-2, a key factor in promoting chondrocyte differentiation and cartilage biosynthesis [39, 14]

Statins also interfere with osteoclast function with suppression of bone resorption and have been shown to promote osteoblast activity through increased production of bone morphogeneic protein-2, Such effects should beneficially modify the subchondral OA bone, already recognized as a potential target for structure modification [40]

\section{CONCLUSION}

It can be concluded that Atorvastatin has beneficial effects and reduced the development of OA lesions in a rat model of $O A$ when given from the first day of surgical induction of OA either by oral, IA or combined routs. Also, it was found that combined oral and IA administration of atorvastatin produced better improvement than oral or IA alone that may be explained by synergism in action of atorvastatin when given by combined oral and IA.

\section{REFERENCES}

1.Bonnet CS and Walsh DA (2005):

Osteoarthritis, angiogenesis and inflammation. Rheumatology; 44:7-16.

\section{Pelletier JM, Tat SK and Pelletier} JP (2010) : Effects of chondroitin sulfate in the pathophysiology of the osteoarthritic joint: a narrative review. Osteoarthritis and-

MANSOURA MEDICAL JOURNAL 
Cartilage;18:7-11

3.David L (2011) : Subchondral bone involvement in the athophysiology of osteoarthritis. Research Signpost; 37/661 (2):69-83.

4.Eglof $C$, Hügle $T$ and Valderrabano V (2012) : Biomechanics and pathomechanisms of osteoarthritis. Swiss Med Wkly; 142:135-183.

5. Zhang $Y$ and Jordan J.M. (2010)

: Epidemiology of osteoarthritis. Clin Geriatr Med; 26:355-369.

6.Goldring M.B. and Goldring S.R. (2010) : Articular Cartilage and Subchondral Bone in the Pathogenesis of Osteoarthritis. Ann N Y Acad Sci; 1192: 230-7.

7. Fu X, Lin L and Zhang $J(2009)$ : Assessment of the Efficacy of Joint Lavage in Rabbits with Osteoarthritis of the Knee. J Orthop Res; 27: 91-96.

8.Ge Z, Hu Y and Heng BC (2006) :
Osteoarthritis and therapy. Arthritis Rheum; 55:493500.

9.Martel-Pelletier J, Wildi LM and Pelletier JP (2012) : Future therapeutics for osteoarthritis. Bone; 51(2): 297-311.

10. Pella D, Rybar R and Mechirova V (2005) : Pleiotropic Effects of Statins. Acta Cardiol Sin; 21(4):190198.

11.Schachter M (2004) : Chemical, pharmacokinetic and pharmacodynamic properties of statins: an update. Fundamental \& Clinical Pharmacology; 19 (1): 117-125 .

12.Waters DD (2005) : Safety of high-dose atorvastatin therapy. Am. J. Cardiol; 96(5): 69-75.

13.Thompson PD, Clarkson $P$ and Karas R (2003) : Statinassociated myopathy. JAMA; 289(13):1681-1690.

14.Lazzerini $P$, Capecchip $P$ and 
Selvi E (2011) : Statins and the Joint: Multiple Targets for a Global Protection? In Seminars in arthritis and rheumatism; 40 (5):430-446.

15.Bendele AM (2001) : Animal models of osteoarthritis. Musculoskelet Neuronal Interact; 1:363-76.

16.Janusz MJ, Bendele AM, Brown KK et al (2002) : Induction of osteoarthritis in the rat by surgical tear of the meniscus: Inhibition of joint damage by a matrix metalloproteinase inhibitor. Osteoarthritis and Cartilage; 10:785-791.

\section{Barsante MM, Roffe E, Yokoro} $\mathrm{CM}$ et al (2005) : Antiinflammatory and analgesic effects of atorvastatin in a rat model of adjuvant- induced arthritis. Eur J Pharmacol; 516:282-289.

18. Dinc M, Sadik M, Kucukalp A, and Faruk $O$ (2012) : An Assessment of the Chondroprotective Effects of In-
tra-Articular Application of Statin and Tetracycline on Early-Stage Experimental Osteoarthritis. International Scholarly Research Network Orthopedics; 2012: 182097.doi:10.5402/2012/ 182097.

19. Damiani $C$, Rosoni $L$ and Vassallo D (2003) : Vasorelaxant effects of eugenol on rat thoracic aorta. Vasuclar Pharmacology; 40: 5966.

20.Beutler E, Duron $O$ and Kelly BM (1963) : Improved method for the determination of blood glutathione. J. Lab. Clin. Med; 61, 882-888.

21.Ohakawa $H$, Ohishi $N$ and Yagi K (1979) : Assay of lipid peroxides in animal tissues by thiobarbituric acid reactions. AnalBiochem; 95 (2):351-358.

22. Papaioannou NA, Triantafillopoulos IK, Khaldi $L$ et al (2007) : Effect of calcitonin in early and late stages of MANSOURA MEDICAL JOURNAL 
experimentally induced osteoarthritis. A histomorphometric study", Osteoarthritis and Cartilage; 15 (4):386-95

\section{Khan HM, Ashraf $M$, Hashmi} AS, Ahmad MD and Anjum AA (2013) : Papain induced degenerative changes in articular cartilage of rat femorotibial joint and its histopathological grading. J. Anim. Plant Sci; 23 (2):350-358.

24.Hochberg $M$, Altman $R$ and Toupin K (2012) : American College of Rheumatology 2012 Recommendations for the Use of Nonpharmacologic and Pharmacologic Therapies in Osteoarthritis of the Hand, Hip, and Knee. American College of Rheumatology; 64: 465-474.

25.Hunter DJ (2011) : Osteoarthritis (Review). Best Pract Res Clin Rheumatol; 25(6):80114.

\section{Akasaki $\mathrm{Y}$, Matsuda $\mathrm{S}$ and $\mathrm{Na-}$}

kayama K (2009) : Mevastatin reduces cartilage degradation in rabbit experimental osteoarthritis through inhibition of synovial inflammation. Osteoarthritis Cartilage; 17:235-43.

27.UlrichVM, Maloney MD and Schwarz EM (2003) : Articular cartilage biology. J Am Acad Orthop Surg; 11 (6):421-30.

28.Wu YS, Hu YY and Yang RF (2007) : The matrix metalloproteinases as pharmacological target in osteoarthritis: statins may be of therapeutic benefit. Med Hypotheses; 69(3):557-9.

29.Rainbow R, Ren W and Zeng $L$ (2012) : Inflammation and Joint Tissue Interactions in OA: Implications for Potential Therapeutic Approaches. Arthritis; 2012 .

30.Alegret, $M$ and Silvestre J (2006) : Pleiotropic effects of statins and related pharmacological experimental approaches. Methods Find 
Exp Clin Pharmacol; 28(9): 627.

31.Abeles $A M$ and Pillinger $M H$ (2006) : Statins as antiinflammatory and immunomodulatory agents: a future in rheumatologic therapy? Arthritis Rheum; 54(2):393407.

\section{Brunella $G$, Lina $R$ and Mauro $F$} (2003) : Enhanced lipid peroxidation in synoviocyte from patient with OA. J. Rheumatol; 30 (2): 345347.

33.Marin V, Profir D, Surdu O, \& Demirgian S (2012) : $\mathrm{Pe}-$ loidotherapy in Osteoarthritis-Modulation of Oxidative Stress. Osteoarthritis - Diagnosis, Treatment and Surgery book edited by Qian Chen, ISBN 978-95351-0168-0. Intech Open Access Publisher.

34.Wagner $\mathrm{AH}$, Kohler T and Ruckschloss U (2000) : Improvement of nitric oxidedependent vasodilatation by HMG-CoA reductase in- hibitors through attenuation of endothelial superoxide anion formation. Arterioscler. Thromb. Vasc. Biol; 20 (1): 61-69.

35.Zhang $H$ and Lin CY (2008) : Simvastatin stimulates chondrogenic phenotype of intervertebral disc cells partially through BMP-2 pathway. Spine; 33(16): 525531.

36.Conaghan PG, Vanharanta H and Dieppe PA (2005) : Is progressive osteoarthritis an atheromatous vascular disease? Ann Rheum Dis; 64:1539-1541.

37.Ndrepepaa $G$, Braunb $S$ and von Beckeratha N (2005) : Oxidized low density lipoproteins, statin therapy and severity of coronary artery disease. Clinica Chimica Acta; 360: 178-186.

38.Violi F, Calvieri C, Ferro D and Pignatelli P (2013) : Statins as Antithrombotic Drugs. American Heart Association;127 (2): 251-257. 
166 POTENTIAL PROPHYLACTIC EFFECT OF ORAL etc...

39.Liao JK and Laufs U (2005) :

Pelletier JP (2010) : Tar"Pleiotropic Effects of Stageting subchondral bone tins," Annual Review Pharmacology \& Toxicology; 45: 89-118.

for treating osteoarthritis: what is the evidence? Best Pract Res Clin Rheumatol;

40. Kwan TS, Lajeunesse D and 24:51-70.

Vol. 44, No. 1 \& 2 Jan. \& April, 2015 


$$
\text { الملحسص العربـى }
$$

\section{تأثير إستخدام الأتور فاستاتين عن طريق الفم}

\section{فى نهـوذج يجريبــى لخشيوذـة المفاصـل}

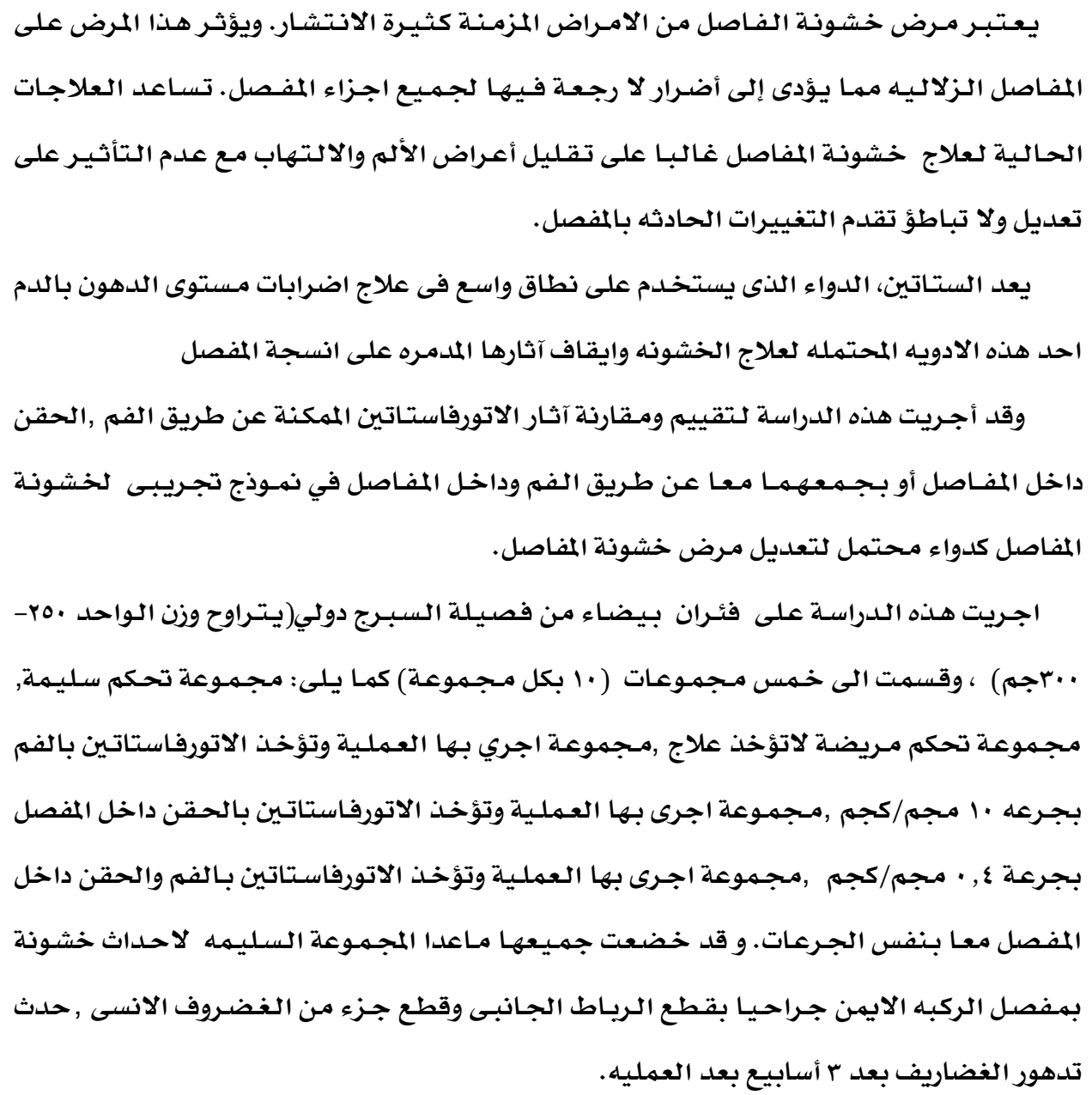


الفه رحقنه داخل المفصل وجمـع تناوله عن طريق الفهم و الحقن داخل المفصل تم أخذ عينات الدم

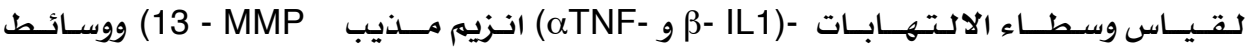

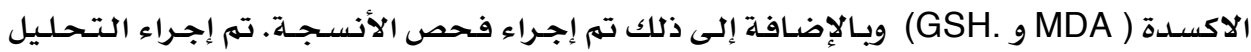

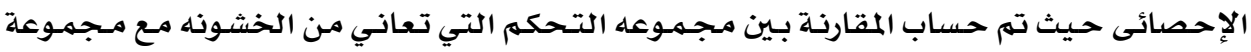

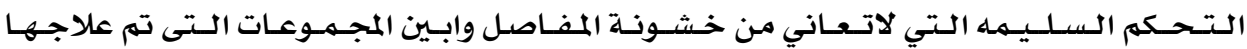

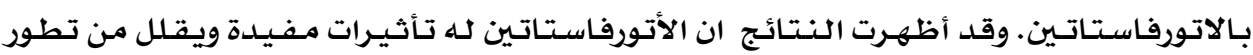
الخـشـونه فى نهـوذج الخـشـونه سـواءا عن طـريق الفهم، الحقن داخل المـفصل أو جـمـع الـطريقـتين معـا.كمـا وجد ان تنـاول ألاتورفاسـتاتين عن طريـق الفهم و الحقن داخل المفـصل معـا أنتجت تحســا أفضل مـن استخدامله بـالفم فقط أو الحقن داخل المفصل فقط. 